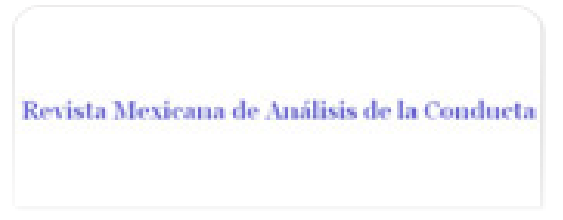

Revista Mexicana de Análisis de la Conducta ISSN: 0185-4534

editora@rmac-mx.org

Sociedad Mexicana de Análisis de la Conducta México

SERRANO, MARIO

COMPLEJIDAD E INCLUSIVIDAD PROGRESIVAS: ALGUNAS IMPLICACIONES Y EVIDENCIAS EMPÍRICAS EN EL CASO DE LAS FUNCIONES CONTEXTUAL, SUPLEMENTARIA Y SELECTORA

Revista Mexicana de Análisis de la Conducta, vol. 35, septiembre, 2009, pp. 161-178

Sociedad Mexicana de Análisis de la Conducta

Guadalajara, México

Disponible en: http://www.redalyc.org/articulo.oa?id=59312304012

Cómo citar el artículo

- Número completo

- Más información del artículo

Página de la revista en redalyc.org

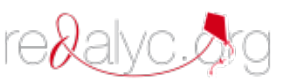

Sistema de Información Científica

Red de Revistas Científicas de América Latina, el Caribe, España y Portugal Proyecto académico sin fines de lucro, desarrollado bajo la iniciativa de acceso abierto 


\title{
COMPLEJIDAD E INCLUSIVIDAD PROGRESIVAS: ALGUNAS IMPLICACIONES Y EVIDENCIAS EMPÍRICAS EN EL CASO DE LAS FUNCIONES CONTEXTUAL, SUPLEMENTARIA Y SELECTORA
}

\author{
PROGRESSIVE COMPLEXITY AND INCLUSIVENESS: SOME \\ IMPLICATIONS AND EMPIRICAL EVIDENCE IN THE CASE OF \\ CONTEXTUAL, SUPPLEMENTARY, AND SELECTOR FUNCTIONS \\ MARIO SERRANO ${ }^{1}$ \\ UNIVERSIDAD VERACRUZANA \\ INSTITUTO DE PSICOLOGÍA Y EDUCACIÓN
}

\begin{abstract}
RESUMEN
Se describen criterios procedurales e índices de ajuste para el análisis experimental y la cuantificación de las funciones contextual (índice de diferencialidad), suplementaria (índice de efectividad) y selectora (índice de precisión) reconocidas en la propuesta taxonómica de Ribes y López (1985). Datos preliminares utilizando ratas como sujetos y un aparato de campo abierto mostraron una alta correspondencia empírica entre los supuestos de complejidad e inclusividad progresivas de dicha propuesta y el ajuste del comportamiento bajo tres tipos de programas de estímulo de entrega de agua: no contingente, contingente y contingente condicional. Específicamente, los índices de diferencialidad fueron más elevados que los índices de efectividad, los que a su vez fueron más elevados que los índices de precisión. Los índices de diferencialidad y efectividad terminales también disminuyeron con los incrementos en la complejidad de los programas para la entrega de agua.
\end{abstract}

1. La correspondencia relacionada con este artículo deberá enviarse al autor a: Universidad Veracruzana, Instituto de Psicología y Educación. Agustín Melgar y Juan Escutia s/n, Colonia Revolución. Xalapa, Veracruz. México, cP 91100.Correo electrónico: marioserrano2003@hotmail.com.

Recibido: 30 de marzo de 2009. Revisado: 17 de junio de 2009. Aceptado: 27 de julio de 2009. 
Palabras clave: psicología interconductual, programas de estímulo, campo abierto, apretar la palanca, ratas.

\begin{abstract}
Procedural criterion and adjustment indexes for the experimental analysis and quantification of contextual (differentiallity index), supplementary (effectiveness index), and selector (precision index) functions recognized in Ribes and López (1985) taxonomic proposal are described. Preliminary data using rats as subjects and an open-field apparatus showed a high empirical correspondence between the taxonomic proposal's progressive complexity and inclusiveness assumptions and the adjustment of behavior under three kinds of stimulus schedules of water delivery: non-contingent, contingent, and conditional-contingent. Specifically, differentiallity indexes were higher than effectiveness indexes, which were higher than precision indexes. Terminal differentiallity and effectiveness indexes also decreased with increases in the complexity of the schedules for water delivery.
\end{abstract}

Keywords: interbehavioral psychology, stimulus schedules, open-field, lever pressing, rats.

En la propuesta taxonómica de Ribes y López (1985), se estima que el comportamiento psicológico puede tener lugar en cinco niveles de organización funcional progresivamente más complejos e incluyentes: a) contextual; b) suplementario; c) selector; d) sustitutivo referencial; y e) sustitutivo no referencial. La diferencia entre estos cinco niveles de organización funcional del comportamiento radica en el criterio de ajuste, la mediación y el nivel de desligamiento funcional implicado en cada uno de ellos. El término criterio de ajuste hace referencia al requerimiento conductual que debe satisfacer el organismo en cada nivel de organización funcional del comportamiento. El término mediación se refiere al proceso por el cual un elemento de estímulo o de respuesta - el mediador - se torna crítico como propiedad estructurante del campo de contingencias. El término desligamiento funcional, por su parte, hace referencia a la relativa autonomía del responder del organismo respecto de las propiedades fisicoquímicas de los eventos de estímulo, así como de los parámetros espaciotemporales que las definen situacionalmente (véase también Carpio, 1994; Ribes, 2004; Ribes, Moreno \& Padilla, 1996).

El desligamiento funcional está posibilitado por la diferenciación sensorial, la motricidad gruesa y fina, la vida en grupo y los ambientes normativos basados en convenciones. En esa medida, es de destacar que mien- 
tras los tres primeros niveles de organización funcional del comportamiento psicológico pueden involucrar organismos no humanos y humanos, los dos últimos niveles involucran organismos humanos exclusivamente debido a que incluyen componentes de estímulo y respuesta de carácter convencional (Ribes \& López, 1985). El presente trabajo abordará los niveles de organización funcional que, de acuerdo con dicha propuesta taxonómica, agotan el comportamiento psicológico de organismos no humanos.

Específicamente, además de describir de una manera relativamente exhaustiva las características de las llamadas funciones contextual, suplementaria y selectora, el objetivo del presente manuscrito es ofrecer una serie de criterios procedurales y de medición para el análisis experimental y la estimación cuantitativa de la configuración de dichos niveles de organización funcional del comportamiento. En la última sección se presentan algunos datos empíricos preliminares, derivados de un proyecto de investigación más general enfocado a contrastar los supuestos de complejidad e inclusividad progresivas que caracterizan a la propuesta taxonómica de Ribes y López (1985). Cabe adelantar que los resultados obtenidos hasta el momento guardan un alto grado de correspondencia con dicha propuesta y sus supuestos.

\section{LAS FUNCIONES CONTEXTUAL, SUPLEMENTARIA Y SELECTORA}

Como se señaló más arriba, en la propuesta taxonómica de Ribes y López (1985) el primer nivel de organización funcional del comportamiento psicológico se denomina función contextual. Este nivel de organización constituye un proceso en el que están implicados por lo menos dos eventos de estímulo (contextualizado y contextualizador) que, debido a sus relaciones de contingencia e inalterables constancias espaciotemporales, deriva en el desligamiento funcional de la respuesta (Ry) respecto de las propiedades filogenéticamente determinadas del evento contextualizado (Ey). En este primer nivel de organización funcional del comportamiento el elemento mediador del campo de contingencias es el evento contextualizador (Ex):

$$
\text { Ex (Ey -----> Ryx) }
$$

El criterio de ajuste implicado en la función contextual es la diferencialidad (Ribes, Moreno \& Padilla, 1996) o la ajustividad (Carpio, 1994), es decir, la adecuación de la actividad del organismo respecto de los parámetros que definen en tiempo y espacio a los eventos contextualizado y contextualizador. De acuerdo con Ribes (2004), la función contextual constituye una relación de isomorfismo en la medida que el organismo reproduce conductualmente alguna o varias de las propiedades del campo de contingencias. En términos 
de la literatura experimental, los fenómenos relativos al condicionamiento clásico (Pavlov, 1927) y la conducta supersticiosa (Skinner, 1948) constituyen algunos ejemplos de la estructuración del comportamiento en el caso de la función contextual.

En el segundo nivel de organización funcional del comportamiento psicológico reconocido en la propuesta taxonómica de Ribes y López (1985), la llamada función suplementaria, el establecimiento de nuevas propiedades funcionales del evento contextualizado depende no únicamente de su relación de contingencia con el evento contextualizador, sino igualmente de un segmento de actividad del organismo (evento suplementario) que media la forma en que entran en relación en tiempo y espacio. En este segundo nivel de organización funcional del comportamiento, el desligamiento de la respuesta (Ryx) es respecto de las regularidades espaciotemporales de los eventos contextualizado y contextualizador:

$$
\text { Ryx (Ey -----> Ex) }
$$

El criterio de ajuste implicado en la función suplementaria es la efectividad, es decir, la adecuación espacial, temporal, topográfica, duracional e intensiva de la actividad del organismo para regular el contacto entre los eventos contextualizado y contextualizador (Carpio, 1994; Ribes, Moreno \& Padilla, 1996). De acuerdo con Ribes (2004), la función suplementaria constituye una relación operativa en la medida que el organismo mediante su actividad, agrega o sustrae componentes del campo de contingencias. La ejecución de los organismos bajo los diversos programas de reforzamiento (Ferster \& Skinner, 1957) y de estímulo (Schoenfeld \& Cole, 1972) en el ámbito del condicionamiento operante, constituyen algunos ejemplos de la estructuración del comportamiento en el caso de la función suplementaria.

El tercer nivel de organización funcional del comportamiento psicológico se conoce bajo el nombre de función selectora. En este caso, la efectividad del evento suplementario para mediar la relación espaciotemporal entre los eventos contextualizado y contextualizador de cuando menos dos campos de contingencias, está mediada a su vez por las propiedades de un evento de estímulo anterior o concurrente denominado evento selector (Es). En esta tercera función, el desligamiento funcional de la respuesta es respecto de las propiedades energéticas absolutas de los eventos contextualizados:

$$
\begin{aligned}
& \text { Es1 ----> (Ey1 ---->> Ryx1 -----> Ex) } \\
& \text { Es2 ----> (Ey2 ---->> Ryx2 ----> Ex) }
\end{aligned}
$$

El criterio de ajuste implicado en la función selectora es la precisión, es decir, la adecuación pertinente de la actividad del organismo respecto de la variabi- 
lidad funcional de los eventos contextualizados debido a su relación con los eventos selectores (Carpio, 1994; Ribes, Moreno \& Padilla, 1996). De acuerdo con Ribes (2004), la función selectora constituye una relación permutativa en la medida que el organismo responde con precisión a una propiedad de estímulo que varía en correspondencia con otro estímulo o sus propiedades. El establecimiento de discriminaciones condicionales mediante cajas de salto (Lashley, 1938) o procedimientos de igualación de la muestra (Cumming \& Berryman, 1965), constituyen algunos ejemplos de la estructuración del comportamiento en el caso de la función selectora.

\section{PROCEDIMIENTOS Y MEDIDAS EN EL ANÁLISIS EXPERIMENTAL DE LAS FUNCIONES CONTEXTUAL, SUPLEMENTARIA Y SELECTORA}

En la propuesta taxonómica de Ribes y López (1985) el término contingencia, entendido como dependencia recíproca entre los elementos involucrados en una interacción y no sólo como su mera contigüidad temporal (e.g. Skinner, 1948), constituye un concepto central. De acuerdo con Ribes (1995), al interior de cualquier nivel de organización funcional del comportamiento psicológico es posible identificar dos tipos de contingencia entre los eventos de estímulo y de respuesta: a) contingencias de ocurrencia; y b) contingencias de función. Las contingencias de ocurrencia determinan la presencia o ausencia de un evento de estímulo o de respuesta en un campo interactivo, mientras que las contingencias de función determinan las propiedades funcionales de tales eventos.

En el caso de la función contextual, por ejemplo, el evento contextualizado es contingente de función al evento contextualizador, es decir, las propiedades psicológicas del primero dependen del segundo. El evento contextualizador es contingente de ocurrencia al evento contextualizado, es decir, la ocurrencia del primero depende de la ocurrencia del segundo. La respuesta al evento contextualizador es contingente de ocurrencia a dicho evento, del mismo modo que la respuesta al evento contextualizado lo es a éste. Finalmente, el evento contextualizador, el evento contextualizado y la respuesta asociada a este último son contingentes de función a la respuesta ante el evento contextualizador.

En el caso de la función suplementaria, el evento contextualizador es contingente de ocurrencia al evento suplementario y el evento contextualizado, del mismo modo que la respuesta al evento contextualizador lo es a éste y al evento suplementario. A su vez, el evento suplementario es contingente de ocurrencia al evento contextualizado. Ambos eventos, suplementario y contextualizado, son contingentes de función tanto al evento contextualiza- 
dor como a la respuesta asociada a éste. Por último, el evento contextualizador es contingente de función a la respuesta asociada a dicho evento.

En el caso de la función selectora, el evento contextualizado es contingente de ocurrencia al evento selector, del mismo modo que el evento suplementario lo es al evento contextualizado. El evento contextualizador y la respuesta asociada a éste son contingentes de ocurrencia al evento suplementario y al evento contextualizado. A su vez, estos últimos son contingentes de función al evento contextualizador y la respuesta asociada a éste. La respuesta al evento contextualizador es contingente de ocurrencia y de función a dicho evento. Finalmente, el evento selector y, de haberla, su correspondiente respuesta, son contingentes de función al evento contextualizador y la respuesta asociada a éste.

Así pues, mientras las contingencias de función engloban las relaciones entre las propiedades circunstanciales de los eventos de estímulo y de respuesta que permiten la evolución de las diferentes funciones psicológicas, las contingencias de ocurrencia se refieren a las condiciones físicas necesarias para que las interacciones puedan tener lugar. A nivel de la exploración empírica, parece importante cuestionarse en torno al procedimiento que permita, por un lado, acoplarse a las características de las diferentes contingencias de ocurrencia que posibilitan el desarrollo de tal o cual nivel de organización funcional del comportamiento psicológico y, por el otro, pueda utilizarse a través de cada uno de ellos a fin de realizar las comparaciones correspondientes intra y entre funciones. En este mismo sentido, la elección del procedimiento también debería contemplar la posibilidad de evaluar el criterio de ajuste implicado en uno u otro nivel de organización funcional del comportamiento. Es posible que los llamados programas de estímulo reseñados por Schoenfeld y Cole (1972) satisfagan dichos requerimientos.

Programas de estímulo

En su versión original, los programas de estímulo consisten en un ciclo repetitivo de tiempo denominado $\mathrm{T}$, compuesto por dos subciclos denominados $t^{D}$ y $t^{\Delta}$. Cada ciclo $T$ comienza con el subciclo $t^{D}$, tiempo discriminativo en el cual la primera respuesta sobre el dispositivo implicado produce la presentación de un segundo evento de estímulo de acuerdo con una probabilidad ( $p$ ) predeterminada. De no ocurrir una respuesta durante el subciclo $t^{\mathrm{D}}$, el evento de estímulo programado para ese ciclo particular no se presenta. En caso contrario, dicho evento se presenta y se deja transcurrir el tiempo restante hasta el inicio del subciclo $t^{\Delta}$, tiempo en el que la probabilidad de que una respuesta produzca la presentación de un evento de estímulo es menor que en el subciclo $t^{D}$ (usualmente $p=0$ ). La proporción de tiempo discriminativo respecto de $\mathrm{T}$ constituye $\mathrm{T}$, parámetro que describe la disponibilidad relativa 
para la presentación de un evento de estímulo en un ciclo T determinado (Schoenfeld, Cumming \& Hearts, 1956).

Como puede notarse a partir de la descripción anterior, en la versión original de los programas de estímulo están presentes las características de las contingencias de ocurrencia implicadas en la configuración de la función suplementaria: al menos dos eventos de estímulo que pueden entrar en relación en medida de la ocurrencia de una respuesta del organismo. A diferencia de los procedimientos comúnmente utilizados (e.g. Ferster \& Skinner, 1957), dado que dicha posibilidad de contacto está restringida en tiempo, los programas de estímulo no demeritan la adecuación temporal del responder. Adicionalmente, en medida de la potencial pérdida de eventos de estímulo derivada de dicha restricción temporal, los programas de estímulo también brindan una posibilidad real de estimar la efectividad del responder del organismo.

El acoplamiento de los programas de estímulo a las características de las contingencias de ocurrencia implicadas en las dos funciones adyacentes a la función suplementaria, no sólo permitiría mantener constantes los valores de los distintos parámetros que componen dichos programas, sino que adicionalmente podría realizarse a partir de modificaciones mínimas. Por ejemplo, en el caso de las contingencias de ocurrencia implicadas en la configuración de la función contextual, basta con presentar los eventos de estímulo independientemente de la actividad del organismo al interior o al final del subciclo $t^{D}$ (e.g. Ribes \& Torres, 1996). En el caso de las contingencias de ocurrencia implicadas en la configuración de la función selectora, puede agregarse un segundo requerimiento de respuesta o bien un segundo evento de estímulo, así como aquellos que fungirán como eventos selectores (e.g. Carpio, Villegas \& Ribes, 1989).

\section{Medidas de logro}

Como se señaló anteriormente, en la propuesta taxonómica de Ribes y López (1985) se estima que en cualquier nivel de organización funcional del comportamiento psicológico está implicado un criterio de ajuste, es decir, un requerimiento conductual que el organismo debe satisfacer y que en medida de su correspondencia con las contingencias de ocurrencia en cuestión, refleja el grado en que los eventos involucrados directamente en la interacción entran en afectación recíproca. De acuerdo con Ribes (2008), el grado en el que se satisfacen los criterios de ajuste implicados en tal o cual nivel funcional de interacción, igualmente brinda el marco de referencia para estimar el resto de las dimensiones del comportamiento psicológico.

Ribes (2004) ha denominado respuesta requerida al segmento de actividad implicado en la satisfacción del criterio de ajuste de un campo de contingencias de tal o cual nivel de organización funcional. La respuesta al evento de estímulo que en términos de ocurrencia depende de la respuesta 
requerida se denomina respuesta contingente. Los segmentos de actividad que facilitan o dificultan la respuesta requerida se denominan respuestas compatibles y respuesta incompatibles, respectivamente. Los segmentos de actividad que no afectan ni facilitan la respuesta requerida se denominan respuestas irrelevantes. En conjunto con las características operativas de los programas de estímulo antes señaladas y, por supuesto, determinadas condiciones aparatológicas, dicha clasificación de propiedades funcionales de respuesta facilita la formulación de índices de ajuste para los tres primeros niveles de organización funcional del comportamiento psicológico reconocidos en la propuesta taxonómica de Ribes y López (1985).

\section{Índice de diferencialidad}

Como se señaló más arriba, en el caso de la función contextual el criterio de ajuste implicado consiste en la adecuación de la actividad del organismo respecto de los parámetros que definen en tiempo y espacio a los eventos contextualizado y contextualizador. En esta medida, una estimación del ajuste o índice de diferencialidad (ID) podría calcularse como el tiempo (T) que el organismo dedica a la respuesta requerida (Rr) (e.g. meter la cabeza en el orificio del dispensador en presencia del agua) relativo al tiempo total de eventos contextualizadores (Ex) (e.g. tiempo total de entrega de agua), menos la proporción del tiempo que el organismo dedica a la respuesta incompatible (Ri) correspondiente (e.g. meter la cabeza en el orificio del dispensador en ausencia de agua) respecto del tiempo total de observación (s). Formalmente tendríamos que:

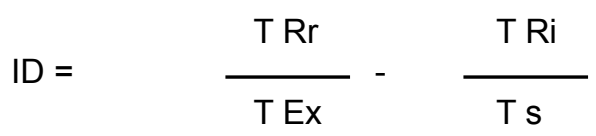

Dependiendo de su valor, el índice señalaría ajuste diferencial incipiente $(0-0.25)$, ajuste diferencial bajo $(0.25-0.5)$, ajuste diferencial medio $(0.5-0.75)$ y ajuste diferencial alto (0.75-1). Valores negativos en el índice de diferencialidad señalarían proporciones de tiempo ocupadas por la Ri superiores a las ocupadas por la $\mathrm{Rr}$, o bien tiempo ocupado por la Ri exclusivamente ${ }^{2}$.

2. Los rangos para determinar los niveles de ajuste están basados en un criterio arbitrario, mientras que la relación sustractiva o multiplicativa entre las razones en cada caso obedece a un criterio empírico. 
Índice de efectividad

En el caso de la función suplementaria, cuyo criterio de ajuste implica la adecuación espacial y temporal de la actividad del organismo para mediar el contacto entre los eventos contextualizado y contextualizador, el índice de efectividad (IE) sería calculado como la proporción de eventos contextualizadores producidos $\left(\mathrm{Ex}_{1}\right)$ respecto de los programados $\left(\mathrm{Ex}_{2}\right)$, multiplicada por el cociente de $E x_{1}$ y la suma de las Rrs (e.g. la primera respuesta sobre la palanca en el subciclo $t^{\mathrm{D}}$ ) y las Ris (e.g. el resto de las respuestas sobre la palanca en el ciclo T) implicadas en su producción. Formalmente se tendría que:

$$
I E=\quad E_{1} \quad x \quad \frac{E x_{1}}{R r+R i}
$$

En línea con los rangos descritos anteriormente para el índice de diferencialidad, dependiendo de su valor el índice señalaría ajuste efectivo incipiente $(0-0.25)$, ajuste efectivo bajo $(0.25-0.5)$, ajuste efectivo medio $(0.5-0.75)$ y ajuste efectivo alto (0.75-1).

Índice de precisión

En el caso de la función selectora, cuyo requerimiento implica la adecuación precisa del responder efectivo respecto de la variabilidad funcional de los eventos contextualizados dado uno u otro evento selector, el índice de precisión (IP) sería calculado como el producto de los cocientes entre las Rrs (e.g. aciertos) y la suma de éstas más las Ris de cada campo contingencial. Dado que en ambos casos las Ris pueden ser por comisión (e.g. errores) o por omisión (i.e. ciclos sin respuesta), formalmente tendríamos que:

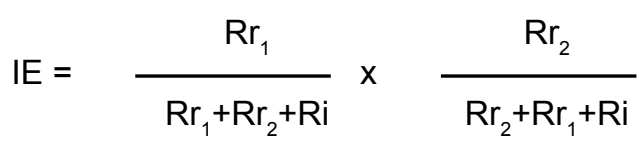

Nuevamente, dependiendo de su valor el índice señalaría ajuste preciso incipiente (0-0.25), ajuste preciso bajo (0.25-0.5), ajuste preciso medio (0.5-0.75) y ajuste preciso alto $(0.75-1)$.

\section{ALGUNAS IMPLICACIONES Y DATOS EMPÍRICOS}

A partir de las reflexiones anteriores en torno a un procedimiento que pueda acoplarse a las contingencias de ocurrencia de las funciones contextual, 
suplementaria y selectora, así como de aquellas relacionadas con la estimación cuantitativa de su configuración, se inició un proyecto de investigación enfocado a contrastar empíricamente los supuestos de complejidad e inclusividad progresivas de las funciones conductuales que caracterizan a la propuesta taxonómica de Ribes y López (1985). Específicamente, sobre ambos supuestos se consideró que:

a) En tanto progresivamente más complejas, la configuración de las funciones contextual, suplementaria y selectora debería tener lugar a ritmos de desarrollo diferentes, delimitados por las características de las contingencias de ocurrencia y los criterios de ajuste implicados en cada una de ellas.

b) Dadas condiciones paramétricas equivalentes, el supuesto de complejidad progresiva implicaría que tanto en el caso de los ritmos de desarrollo como en el de los estados terminales, las funciones más simples deberían superar cuantitativamente a las funciones más complejas.

c) Bajo esas mismas condiciones, el supuesto de complejidad progresiva implicaría que la transición de la función suplementaria a la función selectora debería seguir un ritmo de desarrollo más rápido que la transición de la función contextual a cualquiera de las otras dos funciones superiores, más lento en el caso de la función selectora que en el caso de la función suplementaria.

d) Dado que la inclusión de las funciones más simples dentro de las más complejas supone la subordinación de las primeras respecto de las segundas a partir de la mediación que caracteriza a estas últimas, dicha subordinación debería traducirse en ritmos de desarrollo de la función incluida pautados por los observados para la función incluyente.

e) En medida de la subordinación de las funciones incluidas respecto de los procesos de mediación implicados en las funciones incluyentes, los indicadores cuantitativos de una misma función al nivel de los estados terminales debería disminuir progresivamente entre funciones más complejas, independientemente de la secuencia de exposición a las contingencias de ocurrencia que les caracterizan.

f) Las transiciones descendentes deberían seguir ritmos relativamente similares entre sí, en la medida de que las respuestas requeridas e incompatibles implicadas en las funciones más complejas se tornan irrelevantes en las funciones más simples.

g) El estado de transición ascendente, de tener lugar, estaría caracterizado por un incremento inicial en las respuestas incompatibles de la (s) función (es) incluida (s), cuya disminución sería seguida por un incremento en las respuestas requeridas por la función incluyente.

h) El estado de transición descendente, de tener lugar, estaría caracterizado por un decremento paulatino de las respuestas requeridas e incompatibles implicadas en la (s) función (es) más compleja (s), concomitante al 
incremento progresivo de las respuestas requeridas por la función más simple.

Para confirmar o refutar los puntos anteriores, hasta el momento se han realizado dos estudios de carácter exploratorio y cinco más de corte experimental utilizando un aparto bautizado como caja de Schoenfeld (Ribes, 2008). ${ }^{3}$ Se trata de una cámara de desplazamiento de $92 \mathrm{~cm}$ de largo $\times 92 \mathrm{~cm}$ de ancho x $33 \mathrm{~cm}$ de altura, provista con un dispositivo de video que permite digitalizar la actividad de los sujetos experimentales y, en combinación con un software especializado, segmentarla en periodos de hasta $0.033 \mathrm{~s}$. La imagen digital del piso de la cámara está divida en 64 celdas de $11.5 \mathrm{~cm} \times 11.5 \mathrm{~cm}$. Cada una de las cuatro paredes de la cámara de desplazamiento está constituida por nueve juegos de rieles para la instalación de distintos módulos de estímulo y de respuesta. Tal como se trata de ejemplificar en la figura 1, para la conducción de los estudios antes mencionados, en cada pared de la cámara de desplazamiento se han colocado: a) un dispensador de agua de disponibilidad temporal provisto con un detector de entradas; b) una palanca retráctil a los lados derecho e izquierdo de dicho dispensador y c) tres módulos de estímulo triple (rojo, amarillo y verde) en la parte superior del dispensador.

3. Los estudios se realizaron en el Centro de Estudios e Investigaciones en Comportamiento de la Universidad de Guadalajara con el apoyo y supervisión de Emilio Ribes, quien fungió como tutor del proyecto. 


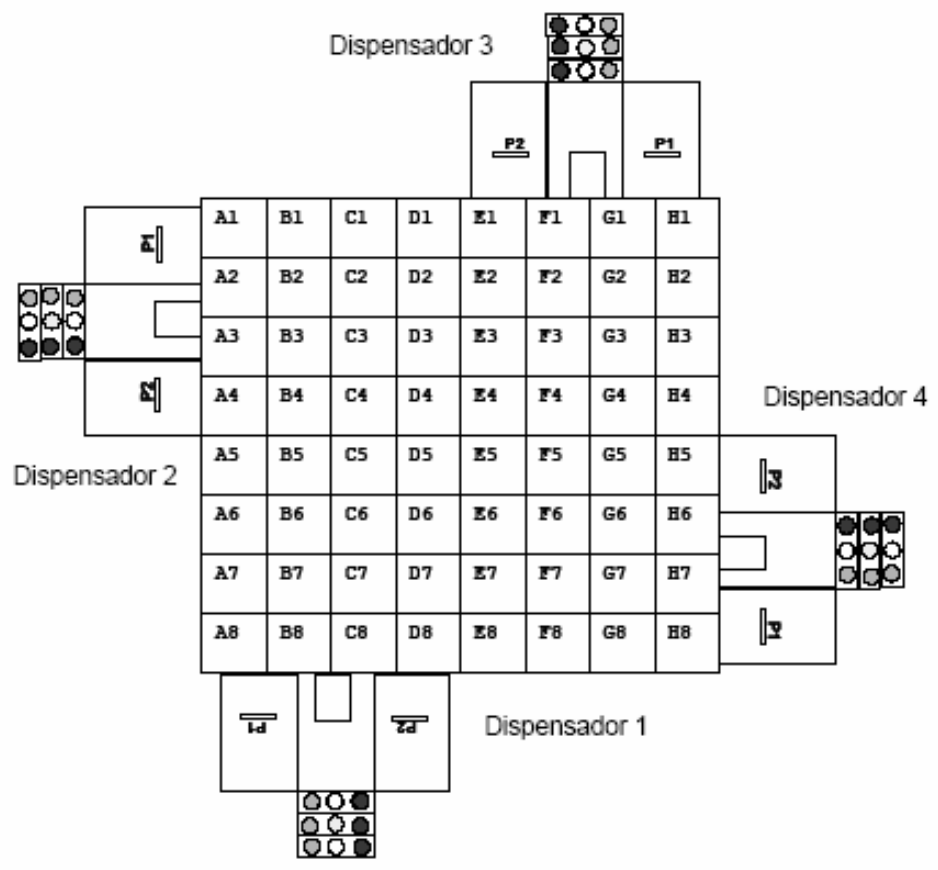

Figura 1. Representación esquemática de la cámara de desplazamiento o "caja de Schoenfeld".

La metodología general de los estudios consistió en exponer a ratas Wistar experimentalmente ingenuas a la entrega no contingente (NK), contingente $(\mathrm{K})$ o contingente condicional $(\mathrm{KC})$ de agua, de acuerdo con tres programas de estímulo independientes y concurrentemente disponibles, compuestos cada uno por 30 ciclos $\mathrm{T}$ y divididos en subciclos $\mathrm{t}^{\mathrm{D}}$ y $\mathrm{t}^{\Delta}$ de $30 \mathrm{~s}$ de duración. Cada programa se correlacionó con uno de tres paneles operativos de la cámara de desplazamiento, mientras en el cuarto panel la actividad de las ratas no tuvo consecuencias programadas. Dependiendo de la condición de entrega de agua, el subciclo $t^{D}$ se señaló mediante los tres leds rojos (NK y $\mathrm{K})$ de cada panel operativo, o bien mediante éstos y los tres leds verdes de manera aleatoria $(\mathrm{KC})$. El subciclo $\mathrm{t}^{\Delta}$ se señaló mediante el led amarillo central exclusivamente.

En la condición $\mathrm{NK}$ el agua se entregó al final del subciclo $\mathrm{t}^{\mathrm{D}}$ independientemente de la actividad de las ratas en los tres paneles operativos de la cámara de desplazamiento. En la condición $\mathrm{K}$ el agua se entregó en uno u 
otro de los tres paneles operativos, como resultado de la primera respuesta al interior del subciclo $t^{\mathrm{D}}$ en cualquiera de las dos palancas ubicadas en cada uno de ellos. En la condición KC el agua también se entregó por la primera respuesta al interior del subciclo $t^{D}$ en una de las dos palancas de los tres paneles operativos; sin embargo, los tres leds rojos o los tres led verdes señalaron ciclo a ciclo la funcionalidad de las palancas izquierda y derecha, respectivamente. Tanto en la condición de entrega de agua $\mathrm{K}$ como en la condición $\mathrm{KC}$, la primera respuesta en el subciclo $\mathrm{t}^{\mathrm{D}}$ también canceló la señal correlacionada con el subciclo.

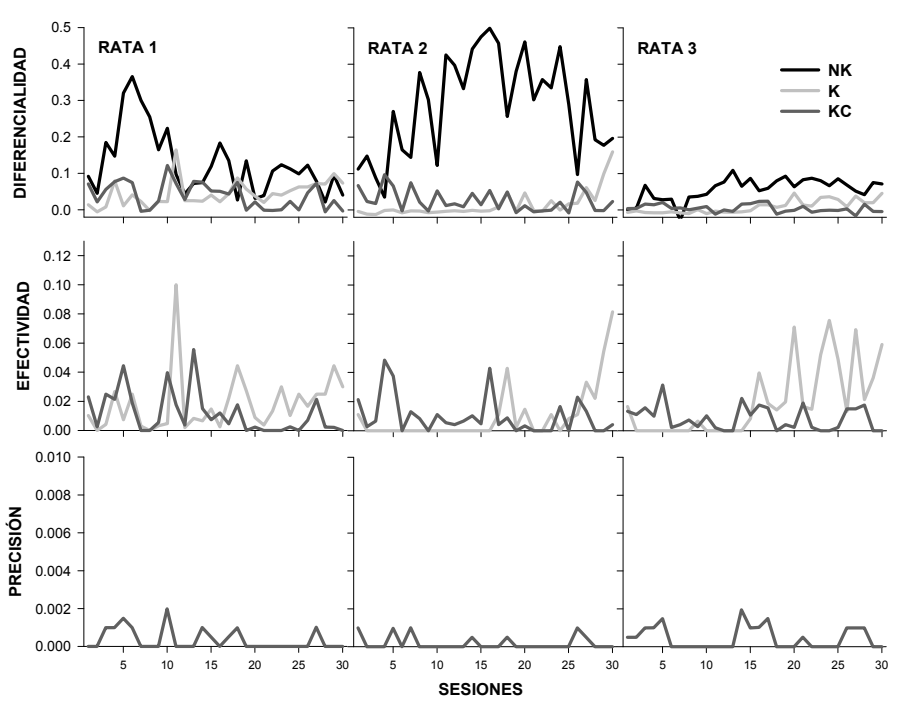

Figura 2. Índices de diferencialidad (paneles superiores), efectividad (paneles centrales) y precisión (paneles inferiores) calculados a lo largo de las condiciones de entrega de agua $N K, K$ y $K C$ para tres ratas.

La figura 2 muestra los índices de diferencialidad (paneles superiores), efectividad (paneles centrales) y precisión (paneles inferiores) calculados para tres ratas expuestas de manera sucesiva a las condiciones de entrega de agua NK, K y KC, en ese orden. Cada condición de entrega de agua estuvo vigente durante 30 sesiones experimentales consecutivas. Nótese que en concordancia con el supuesto de inclusividad progresiva que caracteriza a la propuesta taxonómica de Ribes y López (1985), el índice de diferencialidad (fórmula 1) se calculó en la condición de entrega de agua NK, pero también 
en las condiciones de entrega de agua K y KC. Asimismo, el índice de efectividad (fórmula 2) se calculó en la condición de entrega de agua $\mathrm{K}$ pero también en la condición de entrega de agua KC. El índice de precisión (fórmula 3) se calculó exclusivamente en la condición de entrega de agua KC.

En concordancia con los puntos a y b descritos anteriormente, en la figura 2 se observa que los índices de diferencialidad en la condición de entrega de agua NK fueron superiores a los índices de efectividad en la condición de entrega de agua $\mathrm{K}$, los que a su vez fueron más elevados que los índices de precisión calculados en la condición de entrega de agua KC. Adicionalmente, en línea con el punto c señalado párrafos más arriba, en la figura también se observa que durante las primeras sesiones de la condición de entrega de agua KC, los índices de diferencialidad y efectividad fueron ligeramente más elevados que los calculados en las primeras sesiones de la condición de entrega de agua $\mathrm{K}$. Ello indica que aunque la precisión de la ejecución en la condición de entrega de agua KC fue severamente baja, al inicio de dicha condición las ratas produjeron y bebieron más agua que al inicio de la condición de entrega de agua K. En tercer lugar, en concordancia con el punto $d$ antes mencionado, en la figura 2 se observa que los índices de diferencialidad a lo largo de la condición de entrega de agua $\mathrm{K}$ siguieron ritmos de desarrollo similares a los seguidos por los índices de efectividad a lo largo de dicha condición, mientras que los índices de efectividad a lo largo de la condición de entrega de agua KC siguieron ritmos de desarrollo similares a los seguidos por los índices de precisión. Nótese, sin embargo, que la subordinación de los índices de diferencialidad respecto de los índices de precisión a lo largo de la condición de entrega de agua $\mathrm{KC}$, fue menos evidente que en el caso de los índices de efectividad. 

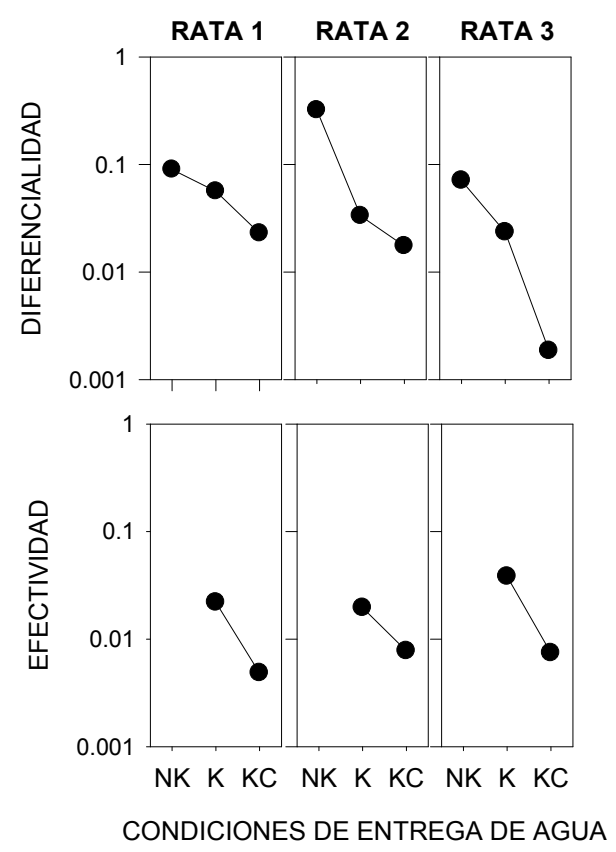

Figura 3. Índices de diferencialidad y efectividad promedio de las últimas 15 sesiones de cada condición de entrega de agua para tres ratas. Nótese la escala logarítmica.

La figura 3 muestra los índices de diferencialidad y efectividad promedio de las últimas 15 sesiones de cada condición de entrega de agua para las mismas tres ratas. En línea con el punto e señalado más arriba, en la figura se observa que los índices de diferencialidad y efectividad al nivel de los estados terminales disminuyeron con el incremento en la complejidad de las contingencias de ocurrencia para la entrega del agua. Dado que estas ratas fueron expuestas a la secuencia NK-K-KC exclusivamente, no fue posible determinar si el decremento progresivo de los índices de diferencialidad y efectividad al nivel de los estados terminales es independiente de la secuencia de exposición a las condiciones de entrega de agua. En esa misma medida, a partir de estos resultados tampoco es posible contrastar empíricamente los puntos $f$ y $h$ antes mencionados. Sin embargo, otros experimentos en los que se manipuló la secuencia de exposición a las condiciones de entrega de agua han mostrado que efectivamente el decremento de los índices de 
diferencialidad y efectividad terminales es independiente de dicha secuencia (Serrano, 2008). Tales estudios han mostrado igualmente que la transición interfuncional ascendente está caracterizada por un incremento inicial de las respuestas incompatibles correspondientes a las funciones incluidas, cuya disminución es seguida por un incremento en las respuestas requeridas por las funciones incluyentes.

\section{CONCLUSIONES}

Después de hacer una revisión de los estudios experimentales en torno de las funciones contextual y suplementaria conducidos hasta ese momento, Ribes y Carpio (1991) concluían que:

Los resultados obtenidos en el programa experimental descrito apuntan a una serie de consideraciones teóricas de interés. Todas ellas, de una u otra manera, se relacionan con la definición de las unidades de análisis de la conducta y los criterios para interpretar la significación de los efectos observados en las condiciones experimentales [...] Quizá sea conveniente volver la vista a los aspectos de precisión funcional de la conducta, en términos de la adecuación temporal de la ejecución a las contingencias que prescriben los programas (Ribes \& Carpio, 1991, pp. 205-208).

Diez años después, en el contexto de algunos datos anómalos derivados del análisis experimental de las funciones contextual y selectora, Carpio, Flores, Bautista, González, Pacheco, Páez y Canales (2001) señalaban que:

[...] la falta de correspondencia entre la evidencia experimental que hemos presentado y los postulados del modelo de Ribes y López (1985) puede ser interpretada no como una prueba en contra de la teoría, tampoco como evidencia de incompetencia metodológica de los autores, sino como un caso que ilustra la inadecuación de la metodología "adoptada" de una teoría ajena con el tipo de evidencia empírica que debería generarse desde la perspectiva interconductual (p. 32).

Es posible que los criterios procedurales y de medición para el análisis experimental y la estimación cuantitativa de la configuración de las funciones contextual, suplementaria y selectora descritos en el presente trabajo, aunados a la utilización de situaciones experimentales con un mayor valor ecológico y la recuperación de la dimensión espacial del comportamiento en términos de su direccionalidad, preferencia, variación y esfuerzo (véase Ribes, 2008), permitan trascender los límites derivados de adoptar la metodología de la teoría del condicionamiento (Ferster \& Skinner, 1957; Skinner, 1938) y, en esa me- 
dida, favorezcan la reflexión en torno a definir nuevas unidades de análisis del comportamiento en correspondencia con los postulados de la psicología interconductual. Los índices de ajuste descritos anteriormente constituyen una primera propuesta en este sentido.

De acuerdo con los resultados presentados, los índices de diferencialidad, efectividad y precisión parecen ser útiles para cuantificar la configuración de las funciones contextual, suplementaria y selectora. Esta "utilidad" obedece no sólo al hecho de que los índices se basen en el criterio de ajuste correspondiente a cada nivel del organización funcional del comportamiento, sino adicionalmente a que en medida de su correspondencia empírica con las implicaciones derivadas de los supuestos de complejidad e inclusividad progresivas de la propuesta taxonómica de Ribes y López (1985), pareciera que efectivamente reflejan y son sensibles a los cambios en el estado de las interacciones. En este sentido, los resultados aquí descritos sugieren que la taxonomía no sólo clasifica de una manera coherente y exhaustiva los fenómenos del comportamiento abordados por distintas escuelas de pensamiento en psicología, sino que adicionalmente su "lógica" y "estructura" guardan un alto grado de correspondencia con dichos fenómenos al nivel de su ocurrencia concreta; al menos en los casos de las funciones evaluadas y bajo los valores paramétricos implementados.

\section{REFERENCIAS}

Carpio, C. (1994). Comportamiento animal y teoría de la conducta. En L. J. Hayes, E. Ribes, \& F. López (Eds.), Psicología interconductual: Contribuciones en honor a J. R. Kantor (pp. 45-68). México: Universidad de Guadalajara.

Carpio, C., Flores, C., Bautista, E., González, F., Pacheco, V., Páez, A. \& Canales, C. (2001). Análisis experimental de las funciones contextual y selectora. En G. Mares \& Y. Guevara (Eds.), Psicología interconductual: avances en la investigación básica (pp. 9-36). México: Universidad Nacional Autónoma de México.

Carpio, C., Villegas, J. \& Ribes, E. (1989). Discriminación condicional en un programa temporal. Revista Mexicana de Análisis de la Conducta, 15, 1-23.

Cumming. W. W. \& Berryman, R. (1965). The complex discriminated operant: Studies of matching-to-sample and related problems. En D. I. Mostofsky (Ed.), Stimulus generalization (pp. 284-330). Stanford: Stanford University Press.

Ferster. C. B. \& Skinner, B. F. (1957). Schedules of reinforcement. Nueva York: Appleton Century Crofts.

Lashley, K. S. (1938). Conditional reactions in the rat. Journal of Psychology, 6, 311324 .

Pavlov, I. P. (1927). Conditioned reflex. Londres: Oxford University Press.

Ribes, E. (2008). Estados y límites del campo, medios de contacto y análisis molar del comportamiento: Reflexiones teóricas. Acta Comportamentalia, 15, 229-259. 
Ribes, E. (2004). Acerca de las funciones psicológicas: Un post-scriptum. Acta Comportamentalia, 15, 117-127.

Ribes, E. (1995). Causalidad y contingencia. Revista Mexicana de Análisis de la Conducta, 21, 133-150.

Ribes, E. \& Carpio, C. (1991). Análisis de los parámetros de estímulo que regulan el comportamiento animal. En V. Colotla (Ed.), La investigación del comportamiento en México (pp. 185-210). México: SMAC, AMC, UNAM, Conacyt.

Ribes, E. \& López, F. (1985). Teoría de la conducta: un análisis de campo y paramétrico. México: Trillas.

Ribes, E. \& Torres, C. (1996). Efectos de la variación en la probabilidad de reforzamiento correlacionada con dos estímulos neutros en un programa definido temporalmente. Revista Mexicana de Análisis de la Conducta, 22, 41-78.

Ribes, E., Moreno, R. \& Padilla, A. (1996). Un análisis funcional de la práctica científica: Extensiones de un modelo psicológico. Acta Comportamentalia, 4, 205-235.

Schoenfeld, W. N., Cumming, W. W. \& Hearst, E. (1956). On the classification of reinforcement schedules. Proceedings of the National Academy of Sciences, 42, 563-570.

Schoenfeld, W. N. \& Cole, B. (1972). Stimulus schedules: The t-tau system. Nueva York: Harper and Row.

Serrano, M. (2008). Análisis experimental de las funciones contextual, suplementaria y selectora: Efectos de la concurrencia y la transición intra e intercontingencial. Tesis doctoral no publicada, Universidad de Guadalajara.

Skinner, B. F. (1938). The behavior of organisms: An experimental analysis. Nueva York: Appleton Century Crofts.

Skinner, B. F. (1948). "Superstition" in the pigeon. The Journal of Experimental Psychology, 38, 168-172. 